\title{
Embryonal tumor with abundant neuropil and true rosettes in the brainstem: case report
}

\author{
Hidetoshi Sato, MD, ${ }^{1}$ Yuzo Terakawa, MD, ${ }^{1}$ Naohiro Tsuyuguchi, MD, ${ }^{1}$ Yuko Kuwae, MD, ${ }^{2}$ \\ Masahiko Ohsawa, MD, ${ }^{2}$ and Kenji Ohata, MD'
}

Departments of ${ }^{1}$ Neurosurgery and ${ }^{2}$ Diagnostic Pathology, Osaka City University Graduate School of Medicine, Osaka, Japan

Embryonal tumor with abundant neuropil and true rosettes (ETANTR) is rarely seen in the brainstem, and there are few case reports of brainstem ETANTR in the literature. Accordingly, the characteristics and the role of surgical treatment of this rare entity remain unclear. The authors present a case of brainstem ETANTR involving a 33-month-old boy along with a review of the literature and discuss the role of surgical removal in the treatment of this entity. In the authors' case, the tumor was surgically treated with subtotal resection, which resulted in improvement of the patient's preoperative symptoms. Chemotherapy was initiated but did not appear to be effective, radiotherapy was declined, and the boy died 6 months after the operation. Based on their analysis of 10 previously reported cases and their own case, the authors conclude that, with respect to survival, surgery may be beneficial even in cases of ETANTR in the brainstem. They note, however, that further studies with a large number of cases are needed to validate the role of surgical treatment in brainstem ETANTR.

http://thejns.org/doi/abs/10.3171/2015.3.PEDS14727

KEY WORDS ETANTR; ETMR; embryonal tumor; brainstem; surgical treatment; oncology

$\mathrm{E}$ MBRYONAL tumor with abundant neuropil and true rosettes (ETANTR) was first reported in 2000 as a rare subtype of embryonal tumor, ${ }^{3}$ and there have been only 80 cases reported in the literature. ETANTR is characterized by the presence of undifferentiated neuroepithelial cells resembling those of a classic primitive neuroectodermal tumor, broad zones of well-differentiated neuropil, and ependymoblastic rosettes arising abruptly from paucicellular regions of neoplastic neuropil. ${ }^{6}$ ETANTR occurs mostly in young children, with a mean age of onset of 24.7 months. The prognosis of this disease is extremely poor, and the overall survival after surgical intervention has been reported to be 13.1 months. ${ }^{2}$ According to a recent literature review, in $70.3 \%$ of all reported cases of ETANTR, the tumors are located in the supratentorial region, and ETANTR in the brainstem is rare, accounting for only $12.5 \%$ of cases. ${ }^{2}$

Here, we describe a case of ETANTR that occurred in the brainstem and was treated with resection. We also discuss the characteristics of this rare entity and review the literature.

\section{Case Report}

History and Presentation

A 33-month-old boy presented with vomiting and a 3 -month history of gait disturbance. He had no specific postnatal or developmental history, and he had begun to walk at the age of 12 months. Neurological examination on admission showed severe truncal ataxia, and he was unable to maintain a sitting posture. In addition, slight left hemiparesis and facial and abducens nerve palsy on the right side were present.

Imaging Findings

Magnetic resonance imaging revealed an intramedul-

ABBREVIATIONS EBL = ependymoblastoma; EMA = epithelial membrane antigen; ETANTR = embryonal tumor with abundant neuropil and true rosettes; ETMR = embryonal tumor with multilayered rosette; FISH = fluorescence in situ hybridization; GFAP = glial fibrillary acidic protein; ICE = a combination of ifosfamide, carboplatin, and etoposide; INI-1 = integrase interactor 1; LIN28A = Lin-28 homolog A; NeuN = neuronal nuclei; PET = positron emission tomography; VDCy = a combination of vincristine, doxorubicin, and cyclophosphamide.

SUBMITTED December 26, 2014. ACCEPTED March 16, 2015

INCLUDE WHEN CITING Published online June 19, 2015; DOI: 10.3171/2015.3.PEDS14727.

DISCLOSURE The authors report no conflict of interest concerning the materials or methods used in this study or the findings specified in this paper. 
lary mass lesion with a maximum diameter of $30 \mathrm{~mm}$ occupying the right dorsal part of the pons. The lesion was compressing the fourth ventricle, leading to obstructive hydrocephalus. The lesion was hypointense on T1weighted imaging and hyperintense on both $\mathrm{T} 2$-weighted imaging and diffusion-weighted imaging (Fig. 1A). Administration of contrast medium resulted in faint enhancement (Fig. 1B). The lesion appeared to have arisen from the brainstem and to have grown exophytically (Fig. 1C). There was no evidence of spinal metastasis on MRI at the time of admission. An ${ }^{11} \mathrm{C}$-methionine positron emission tomography (PET) study showed high accumulation in the lesion with an average lesion-to-normal frontal lobe parenchyma ratio of 1.9 (Fig. 1D).

\section{Operation and Postoperative Course}

Because the patient exhibited progressing symptoms and obstructive hydrocephalus due to the lesion, we performed resection of the intramedullary lesion via a transcerebellomedullary fissure approach. Intraoperatively, the lesion was found to be hemorrhagic but soft enough that internal decompression by means of suction was possible. The border between the lesion and the pons was recognizable, but the upper part of the margin was unclear, resulting in subtotal resection. Postoperative MRI showed improvement of hydrocephalus and subtotal resection of the lesion (Fig. 2). Postoperatively, the patient developed medial longitudinal fasciculus syndrome of the right side, but his preoperative truncal ataxia and right facial nerve palsy improved.
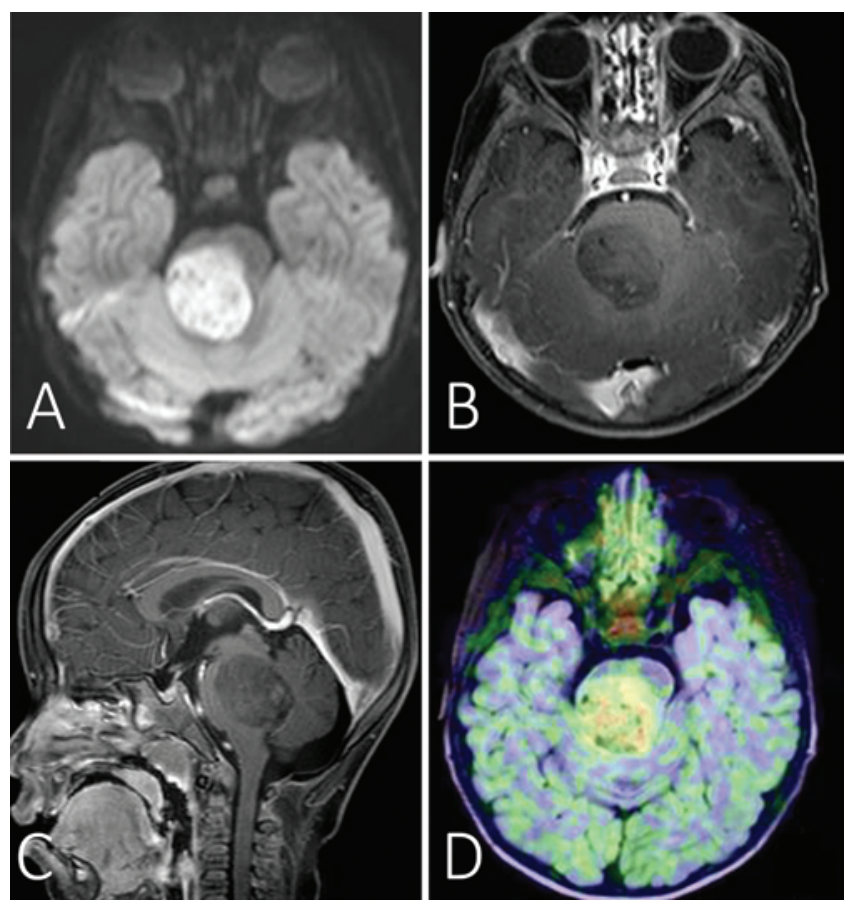

FIG. 1. A: Axial diffusion weighted magnetic MR image showing the the dorsal part of the pons shown as an area of high intensity. B and C: Axial (B) and sagittal (C) T1-weighted MR images demonstrating faint enhancement of the lesion with administration of contrast medium. D: Methionine PET image showing a high accumulation of methionine in the lesion with an average lesion-to-normal frontal lobe parenchyma ratio of 1.9 .
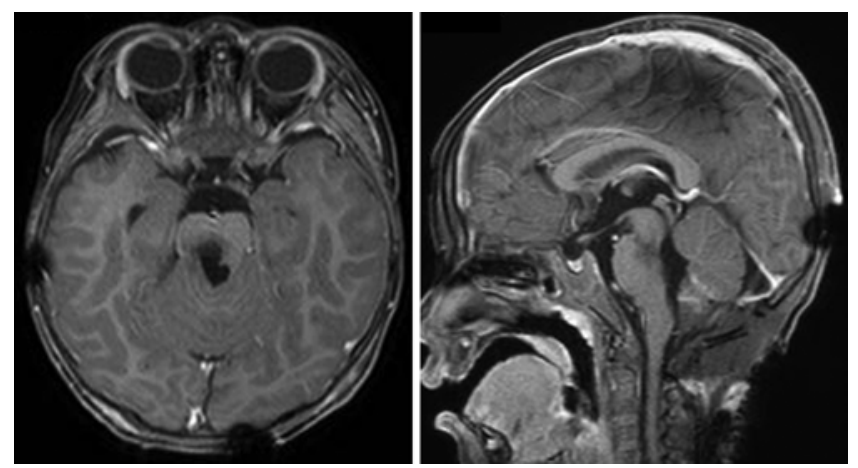

FIG. 2. Postoperative axial (left) and sagittal (right) T1-weighted MR images obtained after administration of contrast medium demonstrating subtotal resection of the lesion.

\section{Pathological Findings}

Histopathological examination revealed the formation of multilayered ependymoblastic true rosettes and some perivascular pseudorosettes, intermingled with neuropilrich stroma containing occasional neurocyte-like cells (Fig. 3A and B). Immunohistochemical staining showed that most of the tumor cells were negative for glial fibrillary acidic protein (GFAP). In contrast, staining for synaptophysin and neurofilament protein was positive in a small population of tumor cells. Staining for neurofilament protein was also positive in neuropil cells. Staining for neuronal nuclei (NeuN) was positive in a small population of tumor cells (Fig. 3C). Staining for epithelial membrane antigen (EMA) was weakly positive in the lumen of the true rosettes (Fig. 3D). Staining for integrase interactor 1 (INI-1) and Lin-28 homolog A (LIN28A) was also positive in most tumor cells (Fig. 3E). The Ki-67 proliferation index was approximately $70 \%$ (Fig. 3F). Based on these pathological findings, the tumor was diagnosed as ETANTR. Furthermore, fluorescence in situ hybridization (FISH) analysis of 19q13.42 also showed amplification of this gene locus, which is a specific molecular marker of ETANTR (Fig. 4).

\section{Adjuvant Therapy and Outcome}

As adjuvant therapy, chemotherapy with a combination of vincristine, doxorubicin, and cyclophosphamide (VDCy) and with a combination of ifosfamide, carboplatin, and etoposide (ICE) was planned. After the first cycle of VDCy and ICE, the tumor continued to grow and spinal dissemination was identified. Therefore, chemotherapy was abandoned. Local radiation with a dose of $36 \mathrm{~Gy}$ and whole-brain and spinal radiation with a dose of $18 \mathrm{~Gy}$ was proposed; however, the parents wished to withdraw treatment, and palliative care was provided. The patient died 6 months after surgery. No autopsy was performed.

\section{Discussion}

In this report, we describe a rare case of ETANTR in the brainstem in which resection was performed. The present case appears to be unique in that methionine PET was used for preoperative evaluation of the tumor and subtotal resection was achieved with minimal neurological deficits. To our knowledge, this is the first case report of ETANTR examined with methionine PET. 

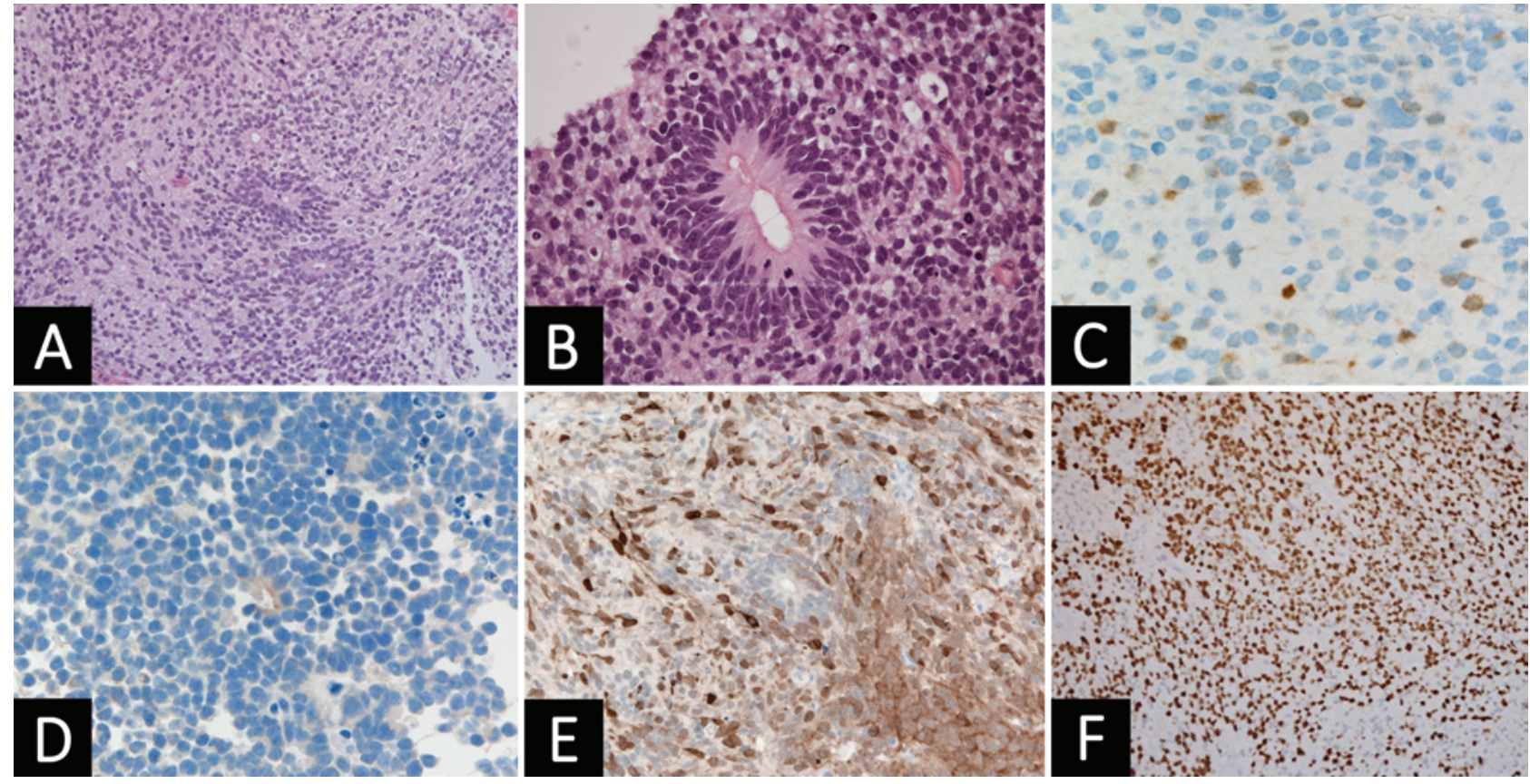

FIG. 3. Histopathological analysis of the lesion. H \& E staining (A and B) demonstrated hypercellular areas that included multilayered ependymoblastic rosettes. A small population of tumor cells was positive for NeuN (C). The lumens of true rosettes were positive for EMA (D). Most tumor cells were positive for LIN28A (E). Ki-67 expression was as high as 70\% (F). Original magnifications $\times 200(A, E$, and $F)$ and $\times 400(B-D)$.

A recent review of 72 cases of ETANTR showed a mean patient age of 24.7 months and a female-to-male ratio of 1.3. The overall survival of patients with this disease is extremely poor, reported to be 13.1 months in this literature review, despite multidisciplinary treatment with surgery, chemotherapy, and radiotherapy. ${ }^{2}$ ETANTR most often affects a supratentorial region, and ETANTR in the brainstem is rare, accounting for only $12.5 \%$ of cases. ${ }^{2}$ To our knowledge, only 11 cases have been reported to date, including our case (Table 1).

The role of surgical treatment for ETANTR in the brainstem remains unclear, although aggressive resection has recently been shown to be associated with better overall survival in malignant gliomas. ${ }^{12}$ A previous literature

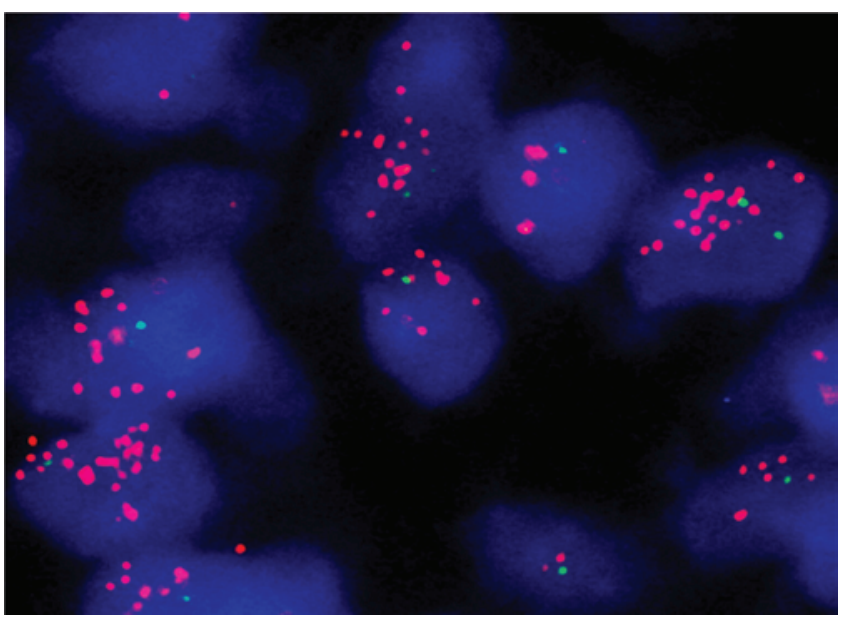

FIG. 4. Fluorescence in situ hybridization (FISH) image demonstrating amplification of the 19q13.42 locus, labeled in pink. review of ETANTR in a variety of locations indicated that patients treated with gross-total or subtotal resection had better survival outcomes than those who underwent only biopsy, but there was no difference in survival between those who underwent gross-total and subtotal resection. ${ }^{2}$ With regard to ETANTR in the brainstem in particular, we constructed Kaplan-Meyer survival curves using our data as well as published survival data. Of 11 cases of ETANTR (Table 1), one was judged to be inoperable and only ventriculoperitoneal shunting and chemotherapy were provided. ${ }^{1}$ In the remaining 10 cases, tumor surgery was attempted, resulting in tumor biopsy in 5 cases $^{6}$ and subtotal or gross-total resection in 5 cases. ${ }^{4,5,7,10}$ The median survival in the 11 reported cases of brainstem ETANTR was 10.2 months -16.4 months in cases in which subtotal or gross-total resection was achieved, but only 4.0 months in the biopsy and inoperative cases. Kaplan-Meyer survival curves stratified with regard to the extent of resection for the 10 cases with available survival data are shown in Fig. 5. Resection of brainstem ETANTR may have a positive impact on overall survival; the log-rank test indicated a difference in these curves, with a probability value of 0.043 . However, we need to interpret this finding with caution because the number of reported cases is small and there may be a selection bias, in that patients with unresectable tumors underwent only biopsy, leading to worse outcomes in those cases. The role of surgery for ETANTR in the brainstem should be determined in future by further studies with a larger number of cases.

ETANTR in the brainstem may have a unique surgical characteristic. La Spina et al. reported a case of ETANTR in the pons in which total surgical removal with preservation of surrounding normal brain tissue was achieved. 
TABLE 1. Summary of reported cases of brainstem ETANTR

\begin{tabular}{|c|c|c|c|c|c|c|c|c|}
\hline Authors \& Year & Age (mos) & Sex & Location & Op & Follow-Up (mos) & CT & $\mathrm{RT}$ & Outcome \\
\hline Fuller et al., 2006 & 48 & M & Pons, midbrain & STR & 19 & Yes & Yes & AWD \\
\hline La Spina et al., 2006 & 48 & M & Pons, midbrain & GTR & 34 & Yes & Yes & NED \\
\hline \multirow[t]{5}{*}{ Gessi et al., 2009} & 22 & $\mathrm{~F}$ & Pons & Biopsy & 7 & Yes & No & DOD \\
\hline & 12 & $\mathrm{~F}$ & Midbrain & Biopsy & 6 & Yes & No & DOD \\
\hline & 18 & $\mathrm{~F}$ & Pons & Biopsy & NA & Yes & No & NA \\
\hline & 16 & $\mathrm{~F}$ & Midbrain & Biopsy & 2 & Yes & No & DOD \\
\hline & 17 & $\mathrm{~F}$ & Brainstem & Biopsy & 2 & Yes & No & DOD \\
\hline Ferri Niguez et al., 2010 & 9 & $\mathrm{~F}$ & Upper brainstem & STR & 5 & Yes & No & DOD \\
\hline Korshunov et al., 2010 & 24 & $\mathrm{~F}$ & Midbrain & STR & 18 & Yes & No & NED \\
\hline Adamek et al., 2013 & 17 & M & Brainstem, pons & None $^{*}$ & 3 & Yes & No & DOD \\
\hline Present case & 33 & M & Pons, midbrain & STR & 6 & Yes & No & DOD \\
\hline
\end{tabular}

AWD = alive with disease $; C T$ = chemotherapy; $D O D=$ dead of disease $; G T R=$ gross-total resection; NA = not available; NED = no evidence of disease; RT = radiotherapy; STR = subtotal resection.

* The tumor was considered inoperable.

They found that the tumor in the brainstem was encapsulated, allowing its successful total removal..$^{10}$ Interestingly, in another case of ETANTR in the pons, autopsy demonstrated a very distinct border between the tumor and adjacent apparently normal tissue. It was suggested that the tumor had grown not in an invasive manner but by expanding within the tissue and consequently that it had compressed the surrounding normal brain tissue. ${ }^{1}$ In our case, there was an apparent border between the tumor and the brainstem, which made aggressive resection possible without causing severe deterioration of neurological functions. Further studies are needed to validate whether this finding is specific to brainstem ETANTR. In particular, autopsies in cases of such rare entities are valuable and would help elucidate the characteristics of ETANTR.

There have been only a limited number of reports of ETANTR, but pathological characteristics have been gradually elucidated. Korshunov et al. used FISH analysis and showed that ependymoblastoma (EBL) and ETANTR have a common genetic signature, i.e., amplification at $19 \mathrm{q} 13.42$ with upregulation of a microRNA cluster. ${ }^{6}$ This genetic anomaly suggests that 3 pathologies previously considered as different tumors, i.e., ETANTR, EBL, and

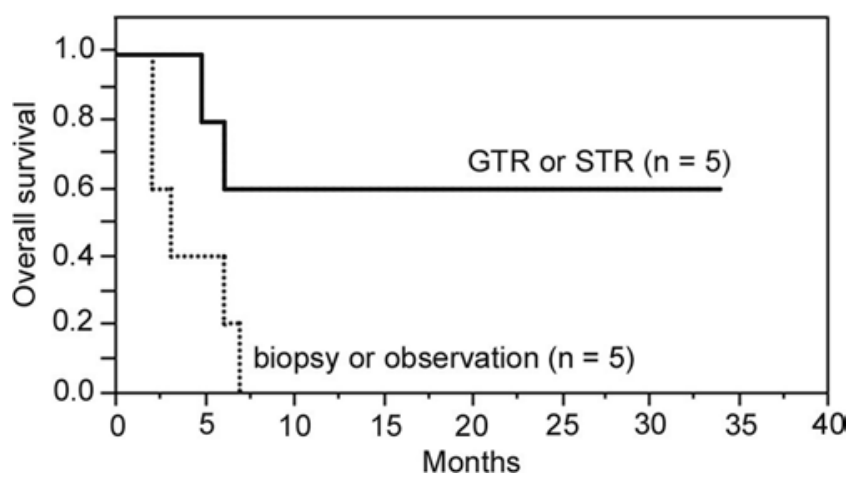

FIG. 5. Kaplan-Meyer survival curve for patients with brainstem ETANTR. Overall survival of the patients in whom gross-total resection (GTR) or subtotal resection (STR) was achieved was better than for those treated with only biopsy or without any operation $(p=0.043)$. medulloepithelioma, may be a single entity proposed as embryonal tumor with multilayered rosette (ETMR).,, 11

Previous molecular analysis of 97 cases of these 3 different pathologies indicated that they have a uniform molecular signature, suggesting that they constitute a single pathological entity. ${ }^{9}$ Moreover, LIN28A immunoreactivity has been reported as a potent diagnostic marker of ETMR. ${ }^{8}$ Therefore, the combination of immunohistochemical staining for LIN28A and FISH analysis of the 19q13.42 locus have been proposed as diagnostic markers of ETMR. ${ }^{9}$ In the present case, both diagnostic markers were positive, consistent with the previous report. ${ }^{9}$

The degree of methionine accumulation on PET in ETANTR remains unclear. We reported a relatively high methionine accumulation with a mean lesion-to-normal parenchyma ratio of 1.9. In general, the methionine accumulation is based on the increased protein synthesis and passive diffusion through the disrupted blood-brain barrier. In the present case, the blood-brain barrier did not appear to be markedly disrupted judging from the degree of contrast enhancement on MRI. Accordingly, the high accumulation seen in this case may have been attributable to the high proliferation rate of tumor cells. In this regard, methionine PET may be useful to predict malignant potential even though a tumor is not enhanced on MRI.

\section{Conclusions}

We have presented a rare case of brainstem ETANTR treated with resection. ETANTR in the brainstem can be resected with minimal morbidity, and therefore surgical removal should be considered as one of the treatment options in these cases. There may be a surgical benefit in terms of survival, but further studies with a large number of cases are needed to validate the role of surgical treatment in ETANTR in the brainstem.

\section{Acknowledgments}

We are grateful to Dr. Junko Hirato of the Department of Pathology, Gunma University Hospital, Maebashi, Japan, and Dr. Sumihito Nobusawa of the Department of Human Pathol- 
ogy, Gunma University Graduate School of Medicine, Maebashi, Japan, for their support in the pathological analyses.

\section{References}

1. Adamek D, Sofowora KD, Cwiklinska M, Herman-Sucharska I, Kwiatkowski S: Embryonal tumor with abundant neuropil and true rosettes: an autopsy case-based update and review of the literature. Childs Nerv Syst 29:849-854, 2013

2. Alexiou GA, Stefanaki K, Vartholomatos G, Sfakianos G, Prodromou N, Moschovi M: Embryonal tumor with abundant neuropil and true rosettes: a systematic literature review and report of 2 new cases. J Child Neurol 28:1709-1715, 2013

3. Eberhart CG, Brat DJ, Cohen KJ, Burger PC: Pediatric neuroblastic brain tumors containing abundant neuropil and true rosettes. Pediatr Dev Pathol 3:346-352, 2000

4. Ferri Niguez B, Martínez-Lage JF, Almagro MJ, Fuster JL, Serrano C, Torroba MA, et al: Embryonal tumor with abundant neuropil and true rosettes (ETANTR): a new distinctive variety of pediatric PNET: a case-based update. Childs Nerv Syst 26:1003-1008, 2010

5. Fuller C, Fouladi M, Gajjar A, Dalton J, Sanford RA, Helton $\mathrm{KJ}$ : Chromosome 17 abnormalities in pediatric neuroblastic tumor with abundant neuropil and true rosettes. Am J Clin Pathol 126:277-283, 2006

6. Gessi M, Giangaspero F, Lauriola L, Gardiman M, Scheithauer BW, Halliday W, et al: Embryonal tumors with abundant neuropil and true rosettes: a distinctive CNS primitive neuroectodermal tumor. Am J Surg Pathol 33:211-217, 2009

7. Korshunov A, Remke M, Gessi M, Ryzhova M, Hielscher T, Witt H, et al: Focal genomic amplification at 19q13.42 comprises a powerful diagnostic marker for embryonal tumors with ependymoblastic rosettes. Acta Neuropathol 120:253260, 2010

8. Korshunov A, Ryzhova M, Jones DT, Northcott PA, van Sluis $\mathrm{P}$, Volckmann R, et al: LIN28A immunoreactivity is a potent diagnostic marker of embryonal tumor with multilayered rosettes (ETMR). Acta Neuropathol 124:875-881, 2012

9. Korshunov A, Sturm D, Ryzhova M, Hovestadt V, Gessi M, Jones DT, et al: Embryonal tumor with abundant neuropil and true rosettes (ETANTR), ependymoblastoma, and medulloepithelioma share molecular similarity and comprise a single clinicopathological entity. Acta Neuropathol 128:279-289, 2013

10. La Spina M, Pizzolitto S, Skrap M, Nocerino A, Russo G, Di Cataldo A, et al: Embryonal tumor with abundant neuropil and true rosettes. A new entity or only variations of a parent neoplasms (PNETs)? This is the dilemma. J Neurooncol 78:317-320, 2006

11. Paulus W, Kleihues P: Genetic profiling of CNS tumors extends histological classification. Acta Neuropathol 120:269-270, 2010

12. Sanai N, Polley MY, McDermott MW, Parsa AT, Berger MS: An extent of resection threshold for newly diagnosed glioblastomas. J Neurosurg 115:3-8, 2011

\section{Author Contributions}

Conception and design: Terakawa, Ohata. Acquisition of data: Sato, Tsuyuguchi. Analysis and interpretation of data: Terakawa, Sato. Drafting the article: Terakawa, Sato, Kuwae. Critically revising the article: Terakawa, Tsuyuguchi, Kuwae, Ohsawa, Ohata. Reviewed submitted version of manuscript: all authors. Approved the final version of the manuscript on behalf of all authors: Terakawa. Statistical analysis: Sato. Administrative/technical/material support: Terakawa, Tsuyuguchi, Kuwae, Ohsawa, Ohata. Study supervision: Terakawa, Tsuyuguchi, Ohata.

\section{Correspondence}

Yuzo Terakawa, Department of Neurosurgery, Osaka City University Graduate School of Medicine, 1-4-3 Asahi-machi, Abenoku, 545-8585 Osaka, Japan. email: terakawa@med.osaka-cu. ac.jp. 